\title{
De nouveaux modèles de soins et de nouvelles compétences sont nécessaires
}

\author{
Les besoins des malades chroniques et des personnes âgées de demain de- \\ mandent de nouveaux modèles de soins, de nouvelles compétences, et une \\ nouvelle répartition de ces compétences («skill-mix») dans les soins de pre- \\ mier recours. Les professions de la santé concernées, médecins et personnel \\ infirmier, devraient relever ces défis conjointement afin de ne pas se perdre \\ dans des luttes de partage.
}

\section{Max Giger, Sabina De Geest}

1 Künzi K, Detzel P. Prise en charge médicale ambulatoire: possibilités de substitution des médecins de premier recours par d'autres groupes professionnels; rapport en allemand, avec résumé en français, basé sur la littérature internationale et des entretiens avec des représentants des groupes professionnels concernés. Neuchâtel: Observatoire suisse de la santé; 2007.

2 Conseil suisse de la science et de la technologie. Démographie médicale et réforme de la formation professionnelle des médecins, 2007. www.swtr.ch (accessed July 17th 2008).

3 Seematter-Bagnoud L, Junod J, Jaccard Ruedin H, Roth M, Foletti C, Santos-Eggimann B. Offre et recours aux soins médicaux ambulatoires en Suisse - projections à l'horizon 2030. (document de travail 33).

Neuchâtel: Observatoire suisse de la santé; 2008

\section{Correspondance:}

Dr Max Giger

Fédération des médecins suisses (FMH)

Elfenstrasse 18

Case postale 170

CH-3000 Berne 15

Tél. 0313591111

Fax 0313591112

max.giger@hin.ch

Prof. Sabina De Geest Institut pour la Science des Soins Infirmiers Université de Bâle Bernoullistrasse 28 CH-4056 Bâle

Tél. 0612670951

Fax 0612670955

sabina.degeest@unibas.ch

\section{Introduction}

Le système de santé actuel, orienté sur les soins aigus, n'est pas équipé pour répondre aux besoins spécifiques des malades chroniques et des personnes âgées. Ce collectif de patients - en forte augmentation par rapport à la population globale - nécessitera une prise en charge renouvelée, à définir dans le cadre de nouveaux modèles de soins respectant les principes du traitement des maladies chroniques et des soins gériatriques. Il faut élaborer rapidement des propositions dans cette perspective.

Pour maîtriser les nouveaux défis thérapeutiques, les professionnels de santé doivent acquérir des compétences leur permettant de travailler de manière efficace dans le cadre de nouveaux modèles de soins médicaux et infirmiers. Ces compétences portent sur l'assistance aux patients dans leur autonomie et sur la collaboration interdisciplinaire. Certes, les modifications de la répartition des compétences, de même que la délégation et la substitution de tâches entre les groupes professionnels de la santé, doivent être analysées d'un œil critique. En égard à l'évolution des besoins, des efforts communs en la matière pourront toutefois contribuer à augmenter la capacité fonctionnelle du système de santé suisse. De nouveaux modèles de coopération peuvent en outre rendre plus attrayante l'activité infirmière ambulatoire et celle de la médecine de famille.

\section{Le système de santé manquera de capacités sous peu}

Au cours des deux dernières années, on a pu lire dans maints rapports et études le souci de leurs auteurs de voir le système de santé (suisse) manquer des moyens nécessaires pour garantir à la population des soins médicaux en quantité suffisante. Ce manque de capacités concerne principa- lement certains aspects des ressources humaines et de la formation en lien avec la future offre de médecins [1-2]. Le dernier rapport de l'Observatoire de la santé «Offre et recours aux soins médicaux ambulatoires en Suisse - projections à l'horizon 2030» [3] fait référence aux défis de demain dans les soins ambulatoires et montre qu'un déséquilibre se produira jusqu'en 2030 entre la demande et l'offre de prestations ambulatoires en médecine de premier recours et en psychiatrie. En raison du vieillissement démographique, le groupe des personnes nécessitant davantage de soins ne cesse de croître et il est considéré comme un facteur important de ce futur déséquilibre.

Pour le corps médical et le personnel infirmier, l'augmentation du nombre de malades chroniques est un défi à maints égards: sous l'angle de l'économie de la santé, on assiste à une rapide expansion d'un segment important du marché; du point de vue de la politique des soins, les structures et processus devront être orientés de manière accrue sur ce segment de la population. Les professions concernées, médecins et personnel infirmier, devraient relever ces défis conjointement afin de ne pas se perdre dans des luttes de partage.

Tout système de santé efficace fournit à la population des prestations adaptées aux besoins de celle-ci [4-5]. De ce fait, l'évolution des besoins de la population en matière de soins, résultant de l'accroissement rapide du nombre d'habitants âgés et très âgés ainsi que de la forte augmentation du nombre des personnes atteintes d'une ou plusieurs maladies chroniques, devrait être l'occasion de repenser la manière de continuer à fournir des prestations de santé efficaces.

En 2050, la part des personnes âgées de 80 ans aura plus que doublé en passant de 5 à $12 \%$, alors que celle des personnes entre 65 et 79 ans aura 
passé de 12 à 16\%. La part des personnes de moins de 39 ans diminuera en corollaire [6]. La probabilité de devoir vivre avec une affection chronique augmente avec l'âge. Actuellement, 80 pour cent des personnes âgées de plus de 65 ans souffrent au moins d'une maladie chronique [7]. Dans les pays industrialisés, il faut s'attendre à ce que la charge globale de morbidité, située en 1990 encore au-dessous de la barre des 50\%, ait augmenté à près de $80 \%$ en 2020 en raison des maladies chroniques [4].

Les besoins en soins des personnes âgées ou souffrant de maladies chroniques se distinguent fortement de ceux des personnes atteintes de maladies aiguës. Alors que le but principal visé pour ces dernières est la guérison, on veille en sonnes âgées et des malades chroniques. Ils ne répondent plus aux exigences, car ils ne tiennent souvent pas suffisamment compte des dimensions psychosociales et comportementales ni du rôle des patients et de leurs proches. La coordination fait défaut. Le suivi thérapeutique, par exemple après un traitement hospitalier, est lacunaire dans bien des cas, la prévention est souvent sacrifiée $[4,5,10,11]$ et les professionnels de santé sont fréquemment engagés de manière inefficace [5].

$\mathrm{Vu}$ les besoins de santé accrus et changeants, le seul fait d'augmenter les ressources en personnel de santé ne pourra pas éviter la pénurie prévisible de soins aux personnes âgées et aux malades chroniques. Les défis liés aux besoins en soins de ce groupe de patients et au manque de
4 World Health Organisation. Innovative care for chronic conditions: building blocks for action. Geneva: WHO; 2002.

5 Institute of Medicine (IOM). Retooling for an Aging America. Building the Health Care Workforce. 2008. www.nap.edu/ catalog/12089.htlm (accessed July 17th 2008).

6 Annuaire statistique de la Suisse. Neuchâtel: Office fédéral de la statistique; 2008.

7 Anderson GF. Medicare and chronic conditions. N Engl J Med. 2005:353:305-9.

8 Moussavi, S, Chatterji S, Verdes E, Tandon A, Patel V, Ustun B. Depression, chronic disease, and decrements in health: Results from the world health surveys. Lancet. 2007;370:851-8.

9 Ciechanowski PS, Katon WJ, Russo JE. Depression and diabetes: Impact of depressive symptoms on adherence, function, and costs. Arch Intern Med. 2000 160(21):3278-85

10 Bodenheimer T. Coordinating care - A perilous journey through the health care system. N Engl J Med. 2008;358:1064-71.

11 Blendon RJ, Schoen C, DesRoches C, Osborn R, Zapert K. Common concerns amid diverse systems: health care experiences in five countries. Health Aff (Millwood). 2003;22(3):106-21.

12 Bodenheimer T, Wagner EH, Grumbach K. Improving primary care for patients with chronic illness. JAMA. 2002;288(14): 1775-9.

13 Bodenheimer T, Wagner EH, Grumbach K. Improving primary care for patients with chronic illness: the chronic care model, Part 2. JAMA 2002;288(15): 1909-14.

\section{L'évolution des besoins de la population en matière de soins appelle le développement de prestations ambulatoires adéquates}

premier lieu, chez les patients âgés, à limiter la progression de la maladie, à prévenir les épisodes aigus, à modifier les facteurs de risque et à procurer une assistance à l'autonomie («self management»). A cet effet, il s'agit de garantir la continuité du traitement entre les médecins et les institutions et de soutenir les personnes concernées dans les activités élémentaires et instrumentales de leur vie quotidienne $[4,5]$. Actuellement déjà, les personnes âgées et les malades chroniques sont soignés majoritairement dans leur environnement habituel, c'est-à-dire à domicile et non dans les EMS ou résidences pour personnes âgées. Par ailleurs, les déménagements dans un EMS ont lieu toujours plus tard, souvent lorsque la personne a un urgent besoin d'aide et de soins.

De surcroît, les maladies chroniques augmentent le risque de graves dépressions, associées à d'importantes atteintes à la santé [8] et à une demande accrue en ressources [9]. Les personnes âgées présentent un risque élevé de blessures (essentiellement par chute), de malnutrition et d'états confusionnels. Les limitations intervenant dans leurs activités quotidiennes élémentaires et instrumentales augmentent le besoin de soins et d'encadrement, et conduisent ainsi à un recours accru aux prestations de santé [5]. En outre, la fourniture de soins à ce groupe de patients nécessite une coordination entre médecins de premier recours, spécialistes, services de soins extrahospitaliers, institutions de soins, pharmacies et établissements de soins aigus et de longue durée [10].

Les systèmes de santé actuels, axés principalement sur les soins aigus, présentent d'importantes lacunes dans la prise en charge des per- relève chez les médecins de premier recours et les psychiatres nécessitent des investissements dans des modèles de soins innovateurs, fondés sur les principes régissant la gestion des maladies de longue durée et les soins aux patients âgés. En outre, compte tenu de l'évolution des besoins, le personnel de santé doit acquérir de nouvelles compétences professionnelles lui permettant de travailler efficacement selon de nouveaux modèles de soins, par exemple des compétences dans la collaboration interdisciplinaire ou dans l'utilisation de technologies de l'information et de la communication. En outre, il s'agit de discuter et de mettre en œuvre des changements dans le dosage des compétences et la délégation des tâches, et probablement aussi dans la manière actuelle d'attribuer et de répartir ces dernières. Ces différents aspects sont présentés plus en détail dans les lignes qui suivent.

\section{Nouveaux modèles de soins pour les malades chroniques et les personnes âgées} Les nouveaux modèles de soins tiennent plus fortement compte des besoins des personnes âgées et des malades chroniques. Les modèles testés à ce jour ont donné de meilleurs résultats que les soins usuels, avec des coûts parfois réduits; d'autres modèles non encore testés paraissent très prometteurs $[4,5,10,12,13]$. Un modèle de soins efficace pour malades chroniques doit intégrer les éléments suivants: 1 . Accès aux soins et continuité de ceux-ci; 2. Participation systématique des patients au processus thérapeutique les concernant; 3. Soutien à l'autonomie du patient et 4 . Coordination des soins entre les structures et 
14 Bergeson SC, Dean JD. A systems approach to patient-centered care. JAMA. 2006;296(23): 2848-51.

15 Kennedy A, Rogers A, Bower P. Support for self care for patients with chronic disease. BMJ 2007; 335(7627):968-70.

16 Committee on Quality of Health Care in America, Institute of Medicine. Crossing the Quality Chasm: A New Health System for the 21st Century. Quality Chasm Series. Washington, DC: Institute of Medicine (IOM); 2001.

17 Jackson S, Morgan G. Change of heart. How a team of North Kirklees primary care trust clinicians used performance management principles to improve coronary heart disease services. Int J Health Care Qual Assur. 2007;20(1): 61-76.

18 Naylor MD, Brooten D, Campbell R, Jacobsen BS, Mezey MD, Pauly MV, et al. Comprehensive discharge planning and home follow-up of hospitalized elders: a randomized clinical trial. JAMA. 1999; 281(7):613-20.

19 Brooten D, Youngblut JM Brown L, Finkler SA, Neff DF, Madigan E. A randomized trial of nurse specialist home care for women with high-risk pregnancies: outcomes and costs. Am J Manag Care. 2001;7(8): 793-803.

20 Brooten D, Naylor MD, York R, Brown LP, Munro BH, Hollingsworth $\mathrm{AO}$, et al. Lessons learned from testing the quality cost model of Advanced Practice Nursing (APN) transitional care. J Nurs Scholarsh. 2002;34(4): 369-75

21 Huss A, Stuck AE, Rubenstein LZ, Egger M, Clough-Gorr KM. Multidimensional preventive home visit programs for communitydwelling older adults: a systematic review and meta-analysis of randomized controlled trials. J Gerontol A Biol Sci Med Sci. 2008;63(3):298-307.

22 Bodenheimer T, Laing BY. The Teamlet model of primary care. Ann Fam Med. 2007;5:457-61.

23 Ferrari A, Rideout B. The collaboration of public health nursing and primary care nursing in the development of a nurse managed health center. Nurs Clin North Am. 2005;40(4):771-8, xii.

les institutions/fournisseurs de prestations [14]. En plus de ces éléments, qui représentent un changement de paradigme dans la fixation des priorités par rapport aux modèles de soins aigus, d'autres aspects sont importants: on citera notamment les investissements dans des systèmes d'information clinique, l'assistance aux professionnels dans la prise de décision, l'utilisation des ressources des communes et les soins interdisciplinaires [4, 5, 12, 13, 15, 16].

Parmi les nouveaux modèles de soins, on trouve par exemple les centres interdisciplinaires de premier recours [17], les modèles de soins intermédiaires appelés «transitional» ou «intermediate care models» $[18,19,20]$, les programmes de visites préventives à domicile pour personnes âgées [21], les modèles «teamlet» [22] et les services de santé dirigés par du personnel infirmier spécialisé [23, 24]. En prévenant une exacerbation des maladies chroniques (broncho-pneumopathie chronique obstructive, insuffisance cardiaque et diabète sucré, entre autres) dans le cadre d'une prise en charge commune et coordonnée par le médecin de famille et le personnel infirmier, on peut notablement réduire les hospitalisations de courte durée et retarder l'entrée dans un foyer médicalisé.

\section{Une collaboration interprofessionnelle visant de meilleurs résultats cliniques est un élément central des modèles de soins à développer}

Il est important que les nouveaux modèles de soins impliquent un changement de paradigme en ce qui concerne les questions suivantes: sur quelles priorités les soins sont-ils orientés? Quelles sont les compétences professionnelles déterminantes pour assurer leur efficacité? Quels sont, à cet effet, les modèles de collaboration nécessaires pour les différents groupes de professionnels de santé? Il incombe aux responsables politiques de créer des conditions générales nécessaires au développement, à la mise en œuvre et au financement des nouveaux modèles de soins et de prise en charge [4].

\section{Quelles nouvelles compétences professionnelles devons-nous acquérir?}

Pour pouvoir répondre aux besoins thérapeutiques des personnes âgées et des malades chroniques et œuvrer efficacement dans le cadre de nouveaux modèles de soins, nous devons acquérir de nouvelles compétences professionnelles. L'OMS a identifié les exigences principales que doivent remplir les professions de la santé au $\mathrm{XXI}^{\mathrm{e}}$ siècle: 1 . Axer les soins sur les patients (or- ganiser les institutions de santé et les structures de soins de manière à systématiquement prendre en compte l'expérience des patients concernant leur maladie); 2. Former des partenariats (capacité à collaborer avec les patients, d'autres fournisseurs de prestations et les communes dans l'intérêt d'une prise en charge efficace); 3 . Améliorer la qualité (concevoir clairement les résultats visés; savoir quels sont les changements susceptibles d'apporter des améliorations et comment évaluer les efforts déployés à cet effet); 4. Maîtriser les technologies de l'information et de la communication (aptitude à utiliser les technologies existantes pour soutenir la prise en charge des patients); 5. Adopter une perspective de santé publique (ne plus se focaliser sur les soins donnés à chaque patient, mais sur la planification de la prise en charge de populations de patients) [25, 26]. Ces composants fondamentaux devraient figurer dans les plans d'étude et les cursus de formation de toutes les professions de la santé.

Dans un rapport publié récemment par l'Institute of Medicine [5], les auteurs soulignent que toutes les professions de la santé devraient disposer d'une formation à la prise en charge des personnes âgées. La Hartford's Foundation et les American Medical Colleges ont développé des 
24 Jones KE, Beeching BA, Roberts P, Devine M, Davies J, Bates CM, et al. Long-term effectiveness of a multifaceted intervention on pain management in a walk-in clinic OJM. 2007;100(4):225-32. Epub $2007 \mathrm{Feb} 22$.

25 Pruitt SD, Epping-Jordan JE. Preparing the 21st century global healthcare workforce. BMJ. 2005 330(7492):637-9

26 World Health Organisation. Preparing a Health Care Workforce for the 21st Century. The Challenge of Chronic Conditions. Geneva: WHO; 2005

27 Leipzig RM. Medical student competencies in geriatric medicine. 2007. www.americangeriatrics. org/newsletter/FIT/fall_2007 story01.asp (accessed August 7, 2008).

28 American Association of Colleges of Nursing (AACN). Nurse Practitioner and Clinical Nurse Specialist Competencies for Older Adult Care. 2004. www.hartfordign. org/case_study/Geriatric\% 20Competencies $\% 20$ for $\%$ 20non-GNPs.pdf (accessed August 7, 2008)

29 Hammick M, Freeth D, Koppel I, Reeves S, Barr H. A best evidence systematic review of interprofessional education: BEME Guide no. 9. Med Teach. $2007 ; 29(8)$ : 735-51.

30 Reeves S, Zwarenstein M, Goldman J, Barr H, Freeth D, Hammick M, et al. Interprofessional education: effects on professional practice and health care outcomes. Cochrane Database Syst Rev. 2008;23;(1):CD002213.

31 De Geest S, Callens B, Gut C, Lindpaintner L, Spirig R. Introducing advanced practice nurses nurse practitioners in health care systems: a framework for reflection and analysis. Swiss Med Wkly. 2008;8(43-44):in press.
Une collaboration interprofessionnelle visant de meilleurs résultats cliniques est un élément central des modèles de soins à développer. L'importance de la formation interprofessionnelle (interprofessional education ou IPE) prégraduée et postgraduée doit être clairement mise en exergue. Ainsi, deux ou plusieurs groupes professionnels apprennent ensemble, et les uns des autres, à améliorer la qualité des soins [29]. Une étude publiée récemment dans la banque de données Cochrane sur l'évaluation de résultats obtenus en formation interprofessionnelle parvient à la conclusion que quatre travaux de synthèse sur six effectués dans une optique IPE présentent des résultats d'apprentissage positifs [30].

\section{Répartition des compétences et partage des responsabilités}

La mise en place de nouveaux modèles de soins n'exige pas seulement l'acquisition de nouvelles compétences, mais aussi une nouvelle distribution des tâches et répartition du travail dans l'intérêt d'une meilleure prise en charge des patients. Pour y parvenir, il faut réévaluer la composition des équipes interdisciplinaires [5]. Ces équipes devraient se distinguer par une répartition optimale des compétences (skill-mix) et un partage des responsabilités dans l'intérêt d'un processus efficace et de bons résultats [31].

La répartition des compétences est donc une notion relativement large, qui fait aussi bien référence à la composition des équipes professionnelles engagées qu'à la délimitation des domaines d'activité et de responsabilité des différentes catégories professionnelles. Des changements de répartition peuvent concerner tant les processus de qualification ultérieurs d'un groupe professionnel déterminé que la substitution des tâches entre différents groupes de professionnels, conduire à une délégation de tâches vers le haut ou vers le bas à l'intérieur d'une échelle hiérarchique unidisciplinaire ou viser des innovations dans cette répartition [32]. Les modifications de répartition des compétences effectuées en vue d'améliorer la capacité fonctionnelle d'un système de santé portent sur un groupe mixte regroupant des médecins et d'autres professionnels de santé, notamment des infirmières et infirmiers ou des équipes de psychiatres et de psychologues cliniques. Des effets positifs peuvent se déployer aux niveaux les plus divers: innovation dans les prestations, remède à la pénurie de personnel dans certains groupes professionnels, p. ex. dans les zones rurales, amélioration de la qualité des soins aux personnes âgées et aux malades chroniques ou augmentation de l'efficacité écono- mique de la fourniture de soins [5, 31, 32, 33].

Les principes de la gestion allégée sont de plus en plus appliqués dans l'industrie de la santé, notamment avec l'introduction des forfaits par cas. Ces principes comprennent une optimisation des processus par l'élimination des inefficacités et des temps morts, l'engagement de personnel disposant d'une formation adéquate conforme aux tâches à remplir, le travail d'équipe et la délégation de responsabilités aux différents acteurs. En bref: la bonne personne au bon moment au bon endroit. De ce fait, nous demandons qu'en vue de répondre aux besoins en soins des personnes âgées et des malades chroniques, on introduise les principes de la gestion allégée («lean management») dans la restructuration des services de santé, principes qui sont déjà appliqués avec succès dans le domaine hospitalier [34]. Pour pouvoir influencer les résultats des soins ambulatoires de manière vraiment positive avec l'application des principes de la gestion allégée à large échelle, il est nécessaire d'introduire un changement de paradigme dans la fourniture des soins et la prise en charge des patients.

Le travail avec les advanced practice nurses (APN) est, par exemple, une voie susceptible de modifier la répartition des compétences dans la foulée du développement de nouveaux modèles de prise en charge. Les APN sont des spécialistes en soins infirmiers qui ont acquis des compétences professionnelles du niveau du master dans le cadre d'une formation postgrade universitaire, souvent simultanément à leur activité professionnelle, aux fins d'assumer des tâches cliniques élargies [31]. Suivant le processus de prise en charge considéré, les APN peuvent assister les médecins dans les soins de premier recours, les compléter ou les remplacer partiellement. A cet égard, les APN sont en mesure d'assumer des tâches ou des prestations qu'ils ou elles n'exécutaient pas auparavant. A l'hôpital, les APN peuvent travailler en étroite collaboration, clairement définie, avec les médecins spécialistes pour les décharger, par exemple dans la formation postgraduée [35]. Leurs activités vont de l'assistance aux patients en matière d'autonomie à la fourniture de soins ainsi qu'à l'application de mesures d'encadrement dans l'environnement social des patients, en passant par le soutien et le contrôle des traitements instaurés; les APN sont également en mesure d'évaluer des symptômes (établissement d'un «diagnostic de travail») et de procéder aux premiers traitements. Les recoupements potentiels de tâches entre médecins et personnel infirmier varient en fonction du système de santé concerné et du domaine de travail concret [31]. 
32 Buchan J, Calman L. Skill-Mix and Policy Change in the Health Workforce. Nurses in Advanced Roles. OECD Health Working Papers No. 17, 2004. doi: $10.1787 / 743610272486$.

33 Ohman-Strickland PA, Orzano AJ Hudson SV, Solberg LI, DiCiccioBloom B, O'Malley D, et al. Quality of diabetes care in family medicine practices: influence of nurse-practitioners and physician's assistants. Ann Fam Med. 2008;6:14-22.

34 Ditzel H. Prozessoptimierung und Servicefreundlichkeit: Was können Krankenhäuser von der Wirtschaft lernen? Hessisches Ärzteblatt. 2007;68(2):77-8.

35 Lundberg S, Wali S, Thomas P, Cope D. Attaining resident duty hours compliance: the acute care nurse practitioners program at Olive View-UCLA Medical Center. Acad Med. 2006;81:1021-5.

36 OECD-Berichte über Gesundheits systeme. Schweiz. Bern: BAG; 2006. Original: OECD Reviews of Health Systems. Switzerland / Examens de l'OCDE des systèmes de santé. Suisse. Paris: OECD 2006.

37 de la Porte PW, Lok DJ, van Veldhuizen DJ, van Wijngaarden J, Cornel JH, Zuithoff NP, et al. Added value of a physician-andnurse-directed heart failure clinic: results from the Deventer-Alkmaar heart failure study. Heart. 2007;93:819-25.

38 Rocha JL. Chronic Diseases: A Challenge for eHealth. In: eHealth Conference 2007 Infrastructures and Health Services. Schriftenreihe der GVG Band 57. Bonn: Nanos; 2008.
Dans le système de santé suisse, où la part des coûts de santé au produit national brut n'est dépassée depuis des années que par les Etats-Unis d'Amérique - et celle des médecins praticiens par habitant $(3,6 / 1000)$ que par la Grèce $(4,4 / 1000)$ et par l'Italie $(4,1 / 1000)[35,36]$ - il faut envisager une délégation de tâches aux APN et au personnel infirmier spécialisé visant essentiellement une prise en charge efficace des patients souffrant de maladies chroniques, telles que par exemple l'insuffisance cardiaque [37] et le diabète sucré [38]. Ce soutien aux médecins de famille pourrait augmenter l'attrait de leur propre profession.

La délimitation entre les prestations des médecins, des APN, des autres spécialistes en soins infirmiers disposant de diplômes de hautes écoles ou de formations particulières et des psychologues cliniques est un «sujet brûlant» dans le pilotage des soins tout au long du traitement devraient ensuite être gérés en fonction des besoins médicaux et infirmiers et des compétences professionnelles nécessaires. La responsabilité du résultat thérapeutique se trouve chez la personne qui a convenu du traitement avec le patient; donc en principe chez le médecin. Il faut s'opposer vigoureusement à ce que cette responsabilité du résultat soit transférée au niveau institutionnel au motif que toute une équipe a participé à son élaboration.

\section{Conclusions}

L'évolution démographique et épidémiologique augmente les besoins en personnel et accélère l'augmentation des coûts de la santé. On ne pourra maîtriser cette évolution que par une gestion rigoureuse des processus et un engagement

\section{En se concentrant sur leur activité médicale proprement dite,} les médecins pourront déléguer davantage à d'autres professionnels de santé spécialisés, notamment aux APN, autres spécialistes en soins infirmiers et psychologues cliniques, certaines activités qu'ils assumaient jusqu'ici

de nombreux domaines de la prise en charge. Tous les groupes professionnels cités bénéficient d'une formation de base et complémentaire pour une activité pratique auprès des patients et devraient pouvoir se concentrer sur ce «travail au front». Les points essentiels de l'activité médicale sont les conseils aux personnes malades et en bonne santé, le diagnostic et les traitements. Les médecins de famille fournissent de nombreuses prestations en-dehors de leur domaine de base, notamment dans le secteur administratif et organisationnel. En se concentrant sur leur activité médicale proprement dite, les médecins pourront déléguer davantage à d'autres professionnels de santé spécialisés, notamment aux APN, autres spécialistes en soins infirmiers et psychologues cliniques, certaines activités qu'ils assumaient jusqu'ici. La continuité dans la prise en charge, tout au long des soins, est l'élément central. Les patients choisissent eux-mêmes leur premier interlocuteur, sous l'impulsion de problèmes de santé généraux et, le plus souvent, des troubles dont ils souffrent. Le suivi de la prise en charge et rationnel des ressources humaines. Il faut créer de nouvelles formes de fourniture de soins. Le nombre croissant de personnes âgées présentant une polymorbidité et souffrant de maladies chroniques nécessite de nouvelles prestations qui, à leur tour, appellent le développement et l'augmentation des compétences professionnelles des spécialistes de santé, avec une adaptation correspondante des cursus de formation de base et postgrade. La mise sur pied d'équipes et la répartition des compétences permettent d'augmenter la qualité et l'efficacité de la fourniture des soins. Pour y parvenir, il faut que les institutions et toutes les professions de la santé mènent un débat franc et approfondi, en s'affranchissant de la défense des intérêts particuliers qui prévaut aujourd'hui. C'est maintenant qu'il faut élaborer des propositions pour résoudre les problèmes de santé du premier tiers du XXI ${ }^{\mathrm{e}}$ siècle. Telle est le défi à relever si nous voulons former de manière bien ciblée les spécialistes nécessaires à l'accomplissement des tâches qui nous attendent. 